\title{
Dritter Teil, Drittes Buch: Der Stern oder die ewige Wahrheit und Tor
}

\author{
Caspar Battegay
}

\section{Fragestellung}

Der letzte Teil des Stern der Erlösung ist nicht etwa mit „Epilog“ oder „Schlusswort" überschrieben, sondern mit dem konkreten Wort Tor. Mit dieser architektonischen Metapher ist das Ende als Ausgang konstruiert. Dementsprechend lauten die letzten Worte von Franz Rosenzweigs Text: „Ins Leben“ (SE 472). Auch typographisch wird diese Konstruktion mit dem Ausdünnen des Drucksatzes nachgeahmt. Das Ende verweist auf das Jenseits des Buches, das durch dieses „Tor“ zu erreichen sei: Das bunte Leben. Das Ende bedeutet Anfang. Doch was bedeutet dieses Paradox?

Dieser Beitrag zum dritten Buch des dritten Teils des Sterns geht von dessen formaler Position als letztem Kapitel aus. Das heisst, es wird zu zeigen versucht, dass es in diesem Buch eine Rhetorik des Finalen, des Letzten, des Schliessens und Über-das-Ende-hinausgehens gibt, die mit Rosenzweigs inhaltlichen Thesen eng verbunden ist. Inhaltlich ist das letzte Kapitel ausdrücklich dem klassischen philosophischen und theologischen Thema der Wahrheit gewidmet. Die Grundfrage „Was ist Wahrheit?" (SE 429) bildet die Leitfrage des letzten Kapitels des Sterns und des Tors. In gewisser Weise ist das Thema der Wahrheit schon von Anfang an im Stern präsent. Dessen

How to cite this book chapter:

Battegay, C. 2021. Dritter Teil, Drittes Buch: Der Stern oder die ewige Wahrheit und Tor. In: Brasser, M., Bojanić, P. and Ciglia, F. P. (eds.) The Star for Beginners: Introductions to the Magnum Opus of Franz Rosenzweig. Pp. 167-183. London: Ubiquity Press. DOI: https://doi.org/10.5334/bco.n. License: CC-BY 
Motto bildet der hebräische Vers aus Psalm 45,5, den Luther mit „Zieh einher der Wahrheit zugut“ und Martin Buber mit „Reite für die Sache der Treue“ übersetzt haben. Die Differenz zwischen den Übersetzungen liegt am unterschiedlichen Verständnis des hebräischen Wortes emeth, das üblicherweise mit Wahrheit übersetzt wird, aber eben auch Festigkeit, Beständigkeit oder Treue bedeuten kann. Am Ende des Sterns nimmt Rosenzweig sein biblisches Motto wieder auf und versucht nun, es auch philosophisch einzubetten. Während die erkenntnistheoretische Frage, ob und wie der menschliche Geist zu sicheren Wahrheiten gelangen kann, in philosophischen Systemen klassischerweise am Anfang steht, beschäftigt sich Rosenzweig erst am Schluss damit. Er bemerkt: „Die Wahrheit aber erscheint immer erst am Ende. Das Ende ist ihr Ort“ (SE 443). Aber was heisst das? Geht es in Rosenzweigs Rede vom Ende um ein Ziel, sei es der Philosophie, der Moral, des Glaubens oder gar des Lebens selbst? Oder ist dieses Ende vielmehr formal auf seine Argumentation bezogen und meint ein Abschliessen des im Text Dargelegten, das Einsetzen eines finalen Schlusssteins im System? Was soll es heissen, dass der „Ort“ der Wahrheit das Ende ist? Und was bedeutet schliesslich Rosenzweigs Emphase des Lebens am Schluss dieses philosophischen Werks, in dessen erstem Satz es heisst, dass jede Erkenntnis mit dem Tod beginnt? („Vom Tode, von der Furcht des Todes, hebt alles Erkennen des All an“ (SE 3)). Was heisst „Leben“ hier eigentlich? Was ist die Stellung dieses Begriffs im System des Sterns und wie sind die Begriffe „Leben“ und „Wahrheit“ mit einander verbunden?

Bevor es hier um diese Fragen gehen kann, also darum, was Rosenzweig über und von der Wahrheit denkt und wie die Wahrheit mit dem zentralen Begriff des Lebens verbunden ist, sollen in einem ersten Teil einige Überlegungen und Verweise zum literarischen und philosophischen Topos des Anfangs im Ende gemacht werden. Diese verschlungene Struktur hat eine lange geistesgeschichtliche Tradition. „Wenn immer auch im philosophischen oder wissenschaftlichen Diskurs von einem Ende die Rede ist [...], markiert diese Rhetorik des Endens zugleich einen weiteren Anfang. Das Wort Finis weist auf diesen ambivalenten Status des Endes hin: das Ende schliesst nicht nur etwas ab (eine Geste, eine Inszenierung, eine Erzählung), es markiert zugleich eine Grenze, einen Übergang. “266 Ähnlich wie das Wort „Finis“, mit dem Johann Wolfgang Goethe das Ende des Faust bezeichnet, bezeichnet auch die Überschrift Tor dieses Oszillieren zwischen Ende und Anfang und in gewisser Weise „das Ende

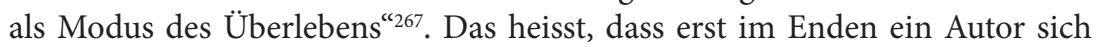
seiner Unsterblichkeit und ein Text sich seines immer wieder aufs Neue zu bewährenden Sinns versichert.

${ }^{266}$ Peter Brandes/Burkhardt Lindner, Einleitung, in: dies. (Hg.), Finis. Paradoxien des Endens, Würzburg 2009, 7-17, hier 9.

${ }^{267}$ Peter Brandes, Rhetorik der Vollendung (Ovid, Goethe), in: Finis. Paradoxien des Endens, 35-50, hier 50. 
Anschaulich wird das, wenn man sich zum Beispiel den sprichwörtlich gewordenen letzten Satz eines berühmten Märchens, des in den Kinder- und Hausmärchen von Jacob und Wilhelm Grimm enthaltenen Dornröschens, in Erinnerung ruft: „, [...] und sie lebten vergnügt bis an ihr Ende. “268 Das Ende der Erzählung stellt kein absolutes Ende dar, sondern den Übergang in das Leben, das nun die in der Geschichte gelernte Moral bei jedem Erzählen neu bewähren muss. Nicht umsonst enden so viele Märchen mit einer Hochzeit, ein Übergangsritual, das Sinn stiftet und in dem gleichzeitig etwas zu einem Ende kommt (das ledige Leben von Braut und Bräutigam) und immer wieder etwas Neues beginnt (das Leben als Paar und die neue Familie).

Letzte Sätze nehmen in vielerlei Hinsicht eine spezielle Position in einem Werk ein. Sie können als Pointe einem Text eine letzte Wendung geben, Sinn festlegen oder problematisch werden lassen. Sie bilden einen Abschied, mit dem ein Autor sich der Nachwelt ins Gedächtnis schreibt, sie besiegeln ein Werk und erklären damit dessen Vollendung oder auch dessen potentielle Unabschliessbarkeit. Jedoch: Einmal ist Schluss. Und auch wenn dieser Schluss ein Abbruch ist, der Linearität des geschriebenen Textes entkommt er kaum. Gerade über die Richtung dieser Linearität hat Rosenzweig nachgedacht und eine landläufige Vorstellung von Aufbau und Entwicklung eines Sinns in einem Text umgekehrt. Im selbstauslegenden Essay Das neue Denken von 1925 bemerkt er, dass in philosophischen Büchern „ein Satz nicht aus seinem Vorgänger“ folge, sondern „viel eher aus seinem Nachfolger” (GS III, 143). Die Schlusssätze nicht nur literarischer, sondern in besonderem Masse philosophischer Werke müssten also besondere Prägnanz aufweisen, vielleicht sogar alles zuvor Stehende fassen, abschliessen, es erklären. Denn wenn sich jeder Satz eines philosophischen Werkes aus seinem nachfolgenden Satz erklären liesse, dann wäre der letzte Satz eben der, bei dem die Erklärungskette stehen bleiben müsste. Der letzte Satz ist der erste, zu dem es keinen nachfolgenden mehr gibt. Das heisst, der letzte Satz würde nicht nur alles Vorherstehende erklären, sondern auch sich selbst.

\section{Vom Ende her denken: Letzte Sätze}

„Als ihm nun schon der Unterleib fast ganz kalt war [sagte er] und das waren seine letzten Worte: O Kriton, wir sind dem Asklepios einen Hahn schuldig, entrichtet ihm den, und versäumt es ja nicht." ${ }^{269}$ So gibt am Schluss von Platons Beschreibung der letzten Stunden des athenischen Philosophen Sokrates dessen Freund und philosophischer Gesprächspartner Phaidon Sokrates' letzte

${ }^{268}$ Jacob und Wilhelm Grimm, Kinder- und Hausmärchen, Ausgabe letzter Hand, München 1977, 284.

269 Platon, Phaidon, übersetzt von Friedrich Schleiermacher und mit einem Nachwort von Andreas Graeser, Stuttgart 1986, 96. 
Worte wieder. Asklepios, dem Gott der Medizin und Heilung, opferte man im antiken Griechenland, wenn man von einer Krankheit genesen war. Sokrates' durch Phaidon und Platon vermitteltes Testament besagt also, dass das Leben selbst eine (vielleicht die) Krankheit darstelle, von der der Tod die Erlösung wäre. In den Tod bedeutet für Sokrates ins Leben. Denn gemäss der im Phaidon dargelegten Philosophie liegt der wahre Grund der Dinge in den Ideen, die ewig sind und dem irdischen Leben nicht unterworfen. Verkürzt argumentiert Platon so: Keine Idee lässt die Verbindung mit dem ihr Entgegengesetzten zu. Auch können Gegenstände, die einen wesentlichen Anteil an einer Idee haben, nicht einen wesentlichen Anteil an einer entgegengesetzten Idee haben. Die Seele ist mit der Idee des Lebens aber wesentlich verbunden, deshalb kann sie nicht teilhaben an der Idee des Todes. Sie ist demnach unsterblich und unvergänglich. Dass Platon am Schluss des Phaidon Sokrates sterben und ihn seinen eigenen Tod als eine Art Genesung kommentieren lässt, besiegelt ähnlich wie die Hochzeit am Schluss des Märchens die philosophische Erzählung mit einer Geste der Bewährung.

Dieses Behaupten der Unsterblichkeit und die Standfestigkeit vor dem Tod begleiten die Philosophiegeschichte, wie es Rosenzweig formuliert, „von Jonien bis Jena" (SE 13), und damit ausdrücklich zu Georg Wilhelm Friedrich Hegels Phänomenologie des Geistes, die in Jena verfasst wurde und 1808 erschien. In der Vorrede zu diesem zentralen Werk des deutschen Idealismus wird der Tod als ,jene Unwirklichkeit“ bezeichnet, der das „Leben des Geistes“ trotzt. ${ }^{270}$ Die letzten Worte von Hegels Phänomenologie bringen dieses Überleben des Geistes als Ziel des Philosophierens überhaupt zum Ausdruck:

Das Ziel, das absolute Wissen, oder der sich als Geist wissende Geist hat zu seinem Wege die Erinnerung der Geister, wie sie an ihnen selbst sind und die Organisation ihres Reiches vollbringen. Ihre Aufbewahrung nach der Seite ihres freien in der Form der Zufälligkeit erscheinenden Daseins ist die Geschichte, nach der Seite ihrer begriffnen Organisation aber die Wissenschaft des erscheinenden Wissens; beide zusammen, die begriffne Geschichte, bilden die Erinnerung und die Schädelstätte des absoluten Geistes, die Wirklichkeit, Wahrheit und Gewissheit seines Throns, ohne den er das leblose Erinnern wäre; nur - aus dem Kelche dieses Geisterreiches schäumt ihm seine Unendlichkeit. ${ }^{271}$

Rein rhetorisch betrachtet, greift Hegel um abzuschliessen zu einem beliebten Trick, nämlich zu einem Zitat, um damit in der Rede eines anderen die eigene $\mathrm{zu}$ bestätigen. Hegel formuliert Friedrich Schillers Die Freundschaft jedoch

${ }^{270}$ Georg Wilhelm Friedrich Hegel, Phänomenologie des Geistes, neu hg. von Hans-Friedrich Wessels und Heinrich Clairmont, mit einer Einleitung von Wolfgang Bonsiepen, Hamburg 1988, 26.

${ }^{271}$ Ebd., 530-531. 
leicht um. Bei Schiller heisst es in der letzten Strophe dieses Gedichts: „Freundlos war der grosse Weltenmeister, / Fühlte Mangel - darum schuf er Geister, / Sel'ge Spiegel seiner Seligkeit! - / Fand das höchste Wesen schon kein Gleiches, / Aus dem Kelch des ganzen Seelenreiches / Schäumt ihm - die Unendlichkeit. “272 Den schönen Gedanken, dass Gott sich die Welt erschuf um endlich Freunde zu finden, säkularisiert Hegel zu einem System, in dem das Abstraktum des „absoluten Geistes“ auftritt, eine Figur, die als Geist sich selbst und seine Geschichte integral begreift und in sich vollständig absorbiert, aufhebt. In Hegels System ist das individuelle Leben und Sterben unwesentlich. Es ist eben das Wesen des Systems, dass seine "Wirklichkeit, Wahrheit und Gewissheit“ darin bestehen, dass jegliche Individualität in der Arbeit am Begriff überwunden werden muss, bis sich das Denken in seiner Gewordenheit selbst begreifend gegenüber steht.

Der Erste Weltkrieg mit all seinen Schrecken und die mit ihm verbundene welthistorische Zäsur leiten den endgültigen Abschied von einer solchen idealistischen Philosophie ein. Den Zeitgenossen erschien es zunehmend absurd, dass angesichts des millionenfachen Abschlachtens mit Giftgas und Maschinengewehr, aber auch angesichts der zunehmenden Globalisierung und Partikularisierung der Gesellschaft so etwas wie ein absoluter Geist sich in „begriffner Geschichte“ spiegeln könnte. Die Geschichte erschien zunehmend nicht als etwas zu Begreifendes, sondern als labyrinthisches Trümmerfeld, dem es irgendwie zu entfliehen gilt. So formuliert Thomas Mann im letzten Satz des 1924 erschienenen Romans Der Zauberberg den Ausgang aus der innerweltlichen Totalität des Todes nur noch als rhetorische Frage nach dessen Möglichkeit: „Wird auch aus diesem Weltfest des Todes, auch aus der schlimmen Fieberbrunst, die rings den regnerischen Abendhimmel entzündet, einmal die Liebe steigen?" ${ }^{\text {“273 }}$ Doch auch die „Liebe“ als Schlagwort, das Weltversöhnung und Transzendenz andeutet, vermag es nicht, den Tod des modernen Menschen zu überdauern. Paradigmatisch bringt diese Skepsis das Werk Franz Kafkas zum Ausdruck. Mit dem vielleicht berühmtesten letzten Satz der deutschen Literatur endet ${ }^{274}$ Der Process, ein Romanfragment, an

${ }^{272}$ Friedrich Schiller, Werke. Nationalausgabe, Band 1, 111.

273 Thomas Mann, Der Zauberberg, Berlin 1924, 938.

${ }^{274}$ Auf dem Deckblatt des Heftes, in das Kafka dieses Kapitel eintrug, schrieb er das Wort „Ende“. Verkompliziert wird für den Process die Rede vom Schluss oder vom letzten Satz allerdings dadurch, dass Kafka nachweislich in seinem Schreibprozess zuerst den Anfang und das Ende geschrieben hatte, um nachher die anderen Kapitel, die teilweise nicht abgeschlossen sind, einzufügen. Die Reihenfolge dieser Kapitel ist bis heute philologisch nicht eindeutig festzulegen, wenn es denn überhaupt je eine gegeben haben sollte. Kafkas Schluss schliesst also nichts ab, sondern legt vielmehr einen Endpunkt fest, zu dem und von dem aus sich verschiedene Textstränge ziehen lassen.Vgl. Roland Reuss, Zur kritischen Edition von „Der Process“ im Rah- 
dem Kafka zwischen 1914 und 1915 geschrieben hat: „,Wie ein Hund!' sagte er, es war, als sollte die Scham ihn überleben. “275

Wenn man es genau nimmt, endet also Kafkas Process, obwohl er mit dem Tod des Protagonisten aufhört, mit dem Wort „leben“. 276 Nur ist dieses Überleben, von dem hier die Rede ist, ein gänzlich anderes als das Überleben des Geistes angesichts des individuellen Todes bei Hegel - und sicher auch ein anderes Leben als das im Stern der Erlösung beschworene. Während bei Hegel das

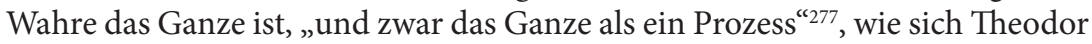
W. Adorno prägnant ausdrückt, präsentiert sich bei Kafka dieses Ganze, dieser Prozess, als dunkle Erfahrung der absoluten, geschlossenen Kontingenz. Denken erlaubt keinen Ausweg aus dieser Krise. Denn der Begriff wird angesichts der Ungeheuerlichkeit der Welt als immer ungemäss empfunden, die Sprache wird auf sich selbst zurückgeworfen. Die Scham ist das Symptom des universellen Scheiterns an Gesetzen, die den Anschein machen, sie seien bloss für den Protagonisten im Augenblick ihres Übertretens geschaffen worden.

Kann es Philosophie so überhaupt noch geben? Ist die Liebe zur Weisheit - und das heisst ja Philosophie - nicht ebenfalls zu einem Ende gekommen, wenn Transzendenz vollkommen von Tod und Scham negiert werden? Diese Frage deutet Thedor W. Adorno an, wenn er sein 1945 erschienenes, bewusst unsystematisches Buch Minima Moralia mit „Reflexionen aus dem beschädigten Leben“ untertitelt. In dem kurzen Text mit dem Titel „Zum Ende“, der als 153. Reflexion Adornos Sammlung von Denkbruchstücken abschliesst, heisst es: „Philosophie, wie sie im Angesicht der Verzweiflung einzig noch zu verantworten ist, wäre der Versuch, alle Dinge so zu betrachten, wie sie vom Standpunkt der Erlösung aus sich darstellten. "278 Gemäss Adorno kann der Gedanke sich nicht von seiner Bedingtheit, der „Entstelltheit und Bedürftigkeit“ lösen, muss jedoch trotzdem den immer scheiternden Versuch unternehmen, diese Unmöglichkeit zu begreifen. „Gegenüber der Forderung, die damit an ihn ergeht“ so lautet der letzte Satz der Minima Moralia, „ist aber die Frage nach der Wirklichkeit oder Unwirklichkeit der Erlösung selber fast gleichgültig. “279

men der Historisch-Kritischen Franz Kafka-Ausgabe, in: Franz Kafka-Hefte 1, Beilage zur Faksimileausgabe des „Process“ in der Historisch-kritischen Ausgabe sämtlicher Handschriften, Drucke und Typoskripte von Franz Kafka, hg. von Roland Reuss und Peter Staengle, Basel/Frankfurt a.M. 1997, 3-25, hier 10.

${ }^{275}$ Franz Kafka, Der Process, Historisch-kritische Ausgabe, Heft „Ende“, 25.

${ }^{276}$ Und ein Blick ins Manuskript des Process zeigt, dass Kafka über-leben trennt, so dass das Wort „leben“ als einziges Wort auf der neuen, letzten Zeile steht.

277 Theodor W. Adorno, Philosophische Terminologie. Zur Einleitung. Band 2, hg. von Rudolf zur Lippe, Frankfurt a.M. 1974, 128.

278 Theodor W. Adorno, Minima Moralia. Reflexionen aus dem beschädigten Leben, Frankfurt a.M. 1980, 333.

${ }^{279}$ Ebd., 334. 
Philosophie vom „Standpunkt der Erlösung“ aus - bietet Der Stern der Erlösung vielleicht eine solche Philosophie an? Wie soll eine solche Philosophie nach dem Zerbrechen des Idealismus beschaffen sein? Und ist auch für Rosenzweig wie für Adorno „die Frage nach der Wirklichkeit oder Unwirklichkeit der Erlösung fast gleichgültig“? Was würde das überhaupt bedeuten? Mit diesen Fragen soll nun zum Schlusskapitel des Sterns und zum Thema der Wahrheit übergeleitet werden.

\section{Wahrheit und Offenbarung}

Rosenzweig ist ebenfalls - wie die erwähnten, genuin modernen Autoren Thomas Mann, Franz Kafka oder Theodor W. Adorno - an jener „Trauerarbeit der

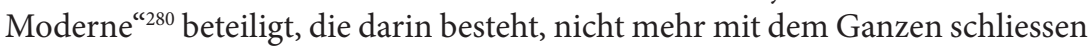
zu können, zu gar keinem schlüssigen Ende eines philosophischen Nachdenkens zu gelangen. Doch gibt er den Anspruch auf dieses Ganze am Ende nicht auf, wenn er auch ganz anders formuliert wird, indem religiöse Kategorien einbezogen werden. Wie Stéphane Mosès festhält, definiert auch Rosenzweig die Wahrheit als das Allumfassende, als das "Ganze des Systems“"281. Aber dieses ist nicht als Logos eines Universal-Ichs verstanden. Die konkrete einzelne Person öffnet sich einer Exteriorität in einer Erfahrung von Passivität, die nichts mit Philosophie und ihrer rationalen Praktiken zu tun hat. Das All, das „einst zerschmettert“, aber „wieder zusammengewachsen“ durch den „Kitt“ der Offenbarung (SE 434). Weiter heisst es:

Das All, das sowohl alles wie ganz wäre, kann weder ehrlich erkannt, noch klar erlebt werden, nur das unehrliche Erkennen des Idealismus, nur das unklare Erleben der Mystik kann sich einreden, es zu erfassen. Das All muss jenseits von Erkenntnis und Erlebnis erfasst werden, wenn es unmittelbar erfasst werden soll. Eben dies Erfassen geschieht in der Erleuchtung des Gebets. (SE 434)

Der Schlussstein des Systems muss somit aus einem Jenseits des philosophischen Diskurses kommen, nämlich aus dem Diskurs der Religion. Dieser Diskurs bildet eine dritte Kategorie neben der rationalen Wissenschaft („Erkenntnis“) und der irrationalen Mystik („Erleben“). Das System übersteigt sich selbst, nicht im Hegelschen Sinn, indem es sich selbst in einer gewaltigen Umstülpungsbewegung begreifen und selbst begründen würde, sondern in einem individuellen Augenblick des Sich-Selbst-Vorfindens in einem Geschaffenen, das Rosenzweig als „Erfassen“ beschreibt. Dieser Moment des Erfassen

${ }^{280}$ Mischa Brumlik, Trauerarbeit der Moderne und melancholischer Messianismus, in: Ludger Heidbrink (Hg.), Entzauberte Zeit. Der melancholische Geist der Moderne, München/Wien: 1997, 210-230.

281 Stéphane Mosès, System und Offenbarung. Die Philosophie Franz Rosenzweigs, München 1985, 210 f. 
verortet er immer als Moment der religiösen Tradition. Es besteht in Rosenzweigs Buch eine schwierige Ambivalenz zwischen einem allegorische und einem konkreten Verständnis: Ist diese religiöse Tradition mit ihren Ritualen, Sprachformeln und -beständen, ihren Narrativen und Feiern, allegorisch, als letztlich doch arbiträre Analogie zu verstehen - oder ist sie ein ontologisch zwangsläufiger Ausdruck eines irgendwo real existierenden Wahren? Diese Frage kann in diesem Beitrag nicht gelöst werden, es kann bloss auf die problematische Stellung des Religiösen im Stern aufmerksam gemacht werden. Auf jeden Fall sind es nun die Möglichkeiten und Grenzen einer Rede über und von Gott, und das Verhältnis von Gott und absoluter Wahrheit, die Rosenzweig am Schluss des Sterns auslotet. Im Hintergrund dieser schwierigen Auslotung stehen die Begriffe der Wahrheit und der Wahrhaftigkeit in Hermann Cohens 1919 ein Jahr nach dessen Tod publiziertem Hauptwerk Religion der Vernunft aus den Quellen des Judentums, auf das sich Rosenzweig immer wieder in ganz unterschiedlicher Weise bezieht. Auf einige dieser Bezüge soll im Folgenden verwiesen werden, wenn auch die Komplexität des Verhältnisses von Rosenzweig zu Cohen es nicht erlaubt, sie hier auszuarbeiten. ${ }^{282}$

Wahrheit wird im letzten Kapitel des Stern der Erlösung in dreifacher Hinsicht verstanden. Erstens entspricht die Wahrheit einer sichtbaren Form, durch die sich das Wesen Gottes offenbart, wie es auch die jüdische Tradition darstellt. $\mathrm{Zu}$ zitieren ist paradigmatisch das Talmudwort, nach dem „das Siegel des Heiligen“ die Wahrheit [hebr. emeth] sei. ${ }^{283}$ Rosenzweig thematisiert hier noch einmal die Kategorie Offenbarung, die für ihn das Verhältnis von Glauben und Wissen, Glauben und Ratio grundlegend verfasst. In der Offenbarung wird die Erfahrung Gottes besiegelt und bewährt - mit Wahrheit versehen. Sie erweist sich als philosophischer und theologischer Begriff, der eine prinzipielle erkenntnistheoretische Stellung und Dignität erhält. Zweitens spricht Rosenzweig damit verbunden von der Wahrheit als einer letzten Wahrhaftigkeit, also dem epistemologischen (=erkenntnistheoretischen) Problem eines letztgültigen Wahrheitskriteriums. Dieses Kriterium ist für Rosenzweig, wie sich zeigen wird, die Faktizität der Wahrheit selbst, die erfahrene oder erfasste Tatsache der Wahrheit. Drittens geht es um die menschliche Erfahrung von Wahrheit in den Religionen. Die Wahrheit ist ein Absolutes, an dem der Mensch immer

${ }^{282}$ Franz Rosenzweig besuchte vor dem Ersten Weltkrieg Kurse bei Cohen an der Hochschule für die Wissenschaft des Judentums in Berlin. Die Beziehung zum 44 Jahre älteren Lehrer war persönlich geprägt, Rosenzweig beschreibt sie in einem Brief als Vater-Sohn-Beziehung. Während er verschiedentlich sehr harsche Kritik an Positionen Cohens übte, bildet doch die Figur Cohens mit der Wendung zur jüdischen Tradition eine fundamentale Bezugsgrösse Rosenzweigs, vgl. Myriam Bienenstock, Cohen face à Rosenzweig. Débat sur la Pensée Allemande, Paris 2009.

${ }^{283}$ Vgl. Sabbat 55a (Der Babylonische Talmud, hg. und übersetzt von Lazarus Goldschmidt, Berlin/Wien 1925, Bd. I, 445.) 
nur Anteil haben kann. Rosenzweig skizziert Judentum und Christentum in ihrem je eigenen anteilmässigen Verhältnis zur Wahrheit. Diese beiden Religionen sind im Stern nicht bloss Religionen. Sie werden vielmehr als die beiden grundsätzlichen Modalitäten menschlicher Existenz verstanden und in ihrem jeweils anderen Verhältnis zur Wahrheit als paradigmatische Seinskategorien beschrieben. ${ }^{284}$ Diese drei verschiedenen Verständnisse von Wahrheit sollen nun eingehend diskutiert werden, bevor zum Schluss auf das Ende und die Bedeutung des Endes für den Stern zurückzukommen ist.

\section{Drei Kontexte von Wahrheit}

A) Gott und Wahrheit. Ist es überhaupt sinnvoll, über Gott zu sprechen? Wer an einen Gott glaubt, bedarf keiner Worte. Gerade das, was sich dem Diskurs entzieht, ist für ihn durch Gott besetzt. Wer aber nicht an Gott glaubt, also wie heute zahlreiche Menschen, für den ist eine solche Rede vorn vorneherein absurd. Es mag darum für viele heutige Leserinnen und Leser die Lektüre des Sterns erschweren, wenn Rosenzweig sich um etwaige Zweifel an der Existenz eines Gottes gar nicht zu kümmern scheint. Doch Rosenzweig nimmt zunächst eine entscheidende Differenzierung vor, die es nicht zu überlesen gilt: Er unterscheidet das Wesen Gottes an sich, und das, was dieses Wesen für uns sein könnte. Zu beachten gilt es den Konjunktiv: „Was Gott, der wahre Gott, vor der Schöpfung gewesen wäre, entzieht sich so jedem Gedanken. Nicht so, was er nach der Erlösung sein würde" (SE 426). Eine Rede von Gott ohne die Menschen, vor der Entstehung der Welt, ist also auch für Rosenzweig sinnlos. Vielmehr möchte er darüber nachdenken, was denn Gott potentiell für uns wäre, wenn eine Erlösung und eine Erlösbarkeit der Welt angenommen wird. Es geht also um eine Rede der Möglichkeit Gottes in einem virtuellen Zustand der Erlösung, gleichsam nach dem Ende der Welt.

Zunächst ist diese Erlösung bloss als eine Art Vorschein, als „Schimmer jenes Augenblicks der göttlichen Liebe“ (SE 424) in der gegenwärtigen Welt präsent.Dieallgemeinerfahrene „Liebe“verbürgedasSein Gottes: „DassGott liebt, erfahren wir, nicht dass Gott die Liebe ist. In der Liebe kommt er uns zu nah, als dass wir noch sagen könnten: dies oder das ist er. Nur dass er Gott ist, erfahren wir in der Liebe, aber nicht, was er ist" (SE 424). Es ist nicht die Prämisse Rosenzweigs, dass aus dem „Weltfest des Todes“ (um nochmals Thomas Mann zu zitieren) die Liebe steigt. Vielmehr macht sich Gott als Herr über Leben und Tod - der weder tot noch lebendig ist, sondern diesen Gegensatz in sich aufhebt - in der Liebe erfahrbar. Der Stern liefert in dem Sinn keine Theodizee (also keine Lösung der Frage, warum das Böse in einer von Gott geschaffenen Welt existiert). Die Grundan-

${ }^{284}$ Vgl. dazu ebenfalls Mosès, System und Offenbarung, 216-220. 
nahme ist vielmehr, dass die Existenz Gottes in der ganz konkreten zwischenmenschlichen Liebe erfahrbar wird, nicht aber das Wesen, nicht das Sein Gottes. Dieses muss wesensmässig verborgen bleiben, und zwar, wie Rosenzweig mit einem für diese Spekulationen typischen Oxymoron festhält, „gerade indem es sich offenbart” (SE 424). Die Offenbarung zeigt dem menschlichen Bewusstsein also nicht das Wesen Gottes. Der denkende Mensch erfährt in der Offenbarung bloss die Präsenz eines sich selbst absolut übersteigenden Anderen. Gott ist diskursiv und bewusstseinsmässig nicht zu fassen. Er ist deshalb auch nicht einfach kongruent mit der Wahrheit an sich. Zwar ist es das Wesen Gottes, wahr zu sein, Gott ist zwar in gewissem Sinn die Wahrheit, doch ist er immer auch mehr. Hermann Cohen, auf den sich Rosenzweig hier implizit bezieht, spricht von der Wahrheit als einzigem „Geltungswert, der dem Wesen Gottes entspricht”285. Die Wahrheit ist bei Cohen das einzige „adäquate Attribut“ Gottes, ohne ihn vollständig beschreiben zu können. Wahrheit steht aber „schon innerhalb der Korrelation zum Menschen, daher kann sie für Gott allein nicht ausdrücklich als Attribut namhaft gemacht werden" ${ }^{286}$. Gott besitzt demnach auch bei Rosenzweig immer eine Art „Überschuss über sein Wesen“ (SE 429). Das bedeutet, dass jegliche Aussagen vom Typus „Gott ist das und das“ nicht gänzlich zutreffend sein können. Rosenzweig fragt aber nicht nur: Was ist Gott? Er fragt auch: Was ist Wahrheit?

B) Tatsächlichkeit der Wahrheit. Rosenzweig verweist zuerst auf die von ihm verworfene idealistische Tradition, nach der die Wahrheit sich selbst verbürgt. Dass es gar keine Wahrheit gebe, könne man aber logisch nicht sagen, denn „mindestens dass es keine Wahrheit gebe, müsste dann wahr sein“ (SE 430). Die Frage, welches „das allgemeine und sichere Kriterium der Wahrheit einer jeden Erkenntnis sei“, stellt Kant in der Kritik der reinen Vernunft. Er differenziert dort zwischen der Wahrheit logischer Urteile, für die das allgemeingültige Kriterium die Übereinstimmung des Urteils mit den Gesetzen des Denkens ist, und der Wahrheit so genannter materialer Urteile, für die es keine allgemeingültigen Kriterien geben kann. ${ }^{287}$ Rosenzweig interessiert sich - und das ist symptomatisch für sein Denken - nicht für diese die Philosophie bis heute prägende Unterscheidung Kants. Für Rosenzweig im Zentrum steht die Beschaffenheit einer transzendentalen Wahrheit, wie sie sich als Faktizität im Leben darstellt. Wahrheit muss gewissermassen zur unbezweifelbaren Tatsache werden. Wie die Offenbarung auch etwas individuell Erfahrenes darstellt, so muss die Gewissheit, dass etwas wahr ist, tatsächlich erfahren werden. Damit

${ }^{285}$ Hermann Cohen, Religion der Vernunft aus den Quellen des Judentums, 2. Aufl. Frankfurt a.M. 1929, 480.

${ }^{286}$ Ebd.

${ }^{287}$ Immanuel Kant, Kritik der reinen Vernunft, nach der ersten und zweiten Originalausgabe hg. von Jens Timmermann, Hamburg 1998, 136. 
setzt er sich vom idealistischen Wahrheitsbegriff ab und tendiert in Richtung eines Existentialismus. So geht es ihm auch nicht um ein Wissen von Wahrheit, sondern um ein Vertrauen darauf: „Auf einem letzten Vertrauen also darauf, dass der Boden, auf den sich die Wahrheit mit ihren eigenen Füssen stellt, tragfähig ist, beruht alles Vertrauen auf die Wahrheit. Die Wahrheit ist selbst der Wahrheit letzte Voraussetzung und ist es nicht als Wahrheit, die auf ihren eigenen Füssen stünde, sondern als Tatsache, der man vertraut. Die Wahrheit selber ist Tatsache noch vor der Tatsache ihrer Unleugbarkeit" (SE 431). Dass die Wahrheit an sich eine Tatsache ist, besagt auch, dass die Wahrheit nicht identisch mit Gott ist, aber „Wahrheit ist von Gott, Gott ist ihr Ursprung“ (SE 432).

Die Erfahrung dieser Tatsächlichkeit sieht Rosenzweig im Gebet und im religiösen Ritus angelegt. Auch hier ist ein Bezug auf Hermann Cohen anzunehmen. Im Kontext seiner Überlegungen zum Gebet meint Cohen: „Gott ist der Gott der Wahrheit, und der Mensch soll der Mensch der Wahrhaftigkeit werden. Darum betet der Mensch zu Gott. “288 Für das Individuum wird die Erfahrung von Wahrheit als Wahrhaftigkeit konkret. Hier ist auch der Grund dafür gelegt, warum für Rosenzweig die Theorie der Wahrheit erst am Schluss seines Systems stehen kann. Wahrheit ist keine abstrakte Idee, keine dem blossen Denken zugängliche Korrelation von Urteil und rationalen Gesetzen. Sinnvoll kann von Wahrheit erst im ethischen Leben, in der Ausgestaltung des individuellen Lebens im religiösen Verband gesprochen werden. Wahrheit muss als Wahrhaftigkeit verbürgt werden, und zwar in der Zentrierung des Lebens auf die Erfahrung der Offenbarung hin. Mit kaum zu überbietendem Pathos schreibt Rosenzweig: „Unser Wahrlich, unser Ja und Amen, mit dem wir auf Gottes Offenbarung antworteten, - es enthüllt sich am Ziel als das klopfende Herz auch der ewigen Wahrheit. Wir finden uns wieder, uns selbst mitten im Brennen des fernsten Sterns der ewigen Wahrheit, nicht die Wahrheit in uns [...], sondern uns in der Wahrheit.“ (SE 436). Dieses „uns in der Wahrheit“ bedeutet, dass die Wahrheit bei Rosenzweig letztlich nicht erkannt werden kann, sondern bewährt werden muss. Die Metapher des klopfenden Herzens besagt, dass Wahrheit nicht ideell gedacht wird, sondern als etwa Lebendiges. Wahrheit selbst ist also ein Prozess: Im aktiven, individuellen Nachvollzug wird Wahrheit erst zur Wahrheit gemacht, von jedem einzelnen immer wieder. Das Paradox ist nun, dass die absolute und einzigartige Wahrheit in verschiedener Weise und in verschiedenen Formen erlebt und bewährt wird. Es gibt in der menschlichen, pluralen Erlebniswirklichkeit kein objektives Kriterium, das die Entscheidung für eine der verschiedenen, immer absoluten Wahrheiten verbürgen würde.

C) Die menschliche Erfahrung von Wahrheit. Ganz entgegen etwa der hegelschen Rede von Wahrheit als dem Ganzen, ist es im Stern gerade die

${ }^{288}$ Cohen, Religion der Vernunft, 443. 
Partikularität, das immer nur Teilhafte, das die Wahrheit ausmacht. Es ist das Wesen der Wahrheit, „zu teil zu sein“. Eine Wahrheit, „die niemandes Teil ist“, ist keine Wahrheit. Auch wenn man eine integrale, absolute Wahrheit annimmt, bildet diese paradoxerweise einen Teil, nämlich einen Teil Gottes, der immer mehr ist als jede Wahrheit (SE 462). Diese Theorie erinnert an die in der berühmten Ringparabel in Gotthold Ephraim Lessings Drama Nathan der Weise (1779) verbildlichte Idee der Toleranz aufgrund der unmöglichen Totalität eines Wahrheitsanspruchs menschlicher Religionen, die aber dennoch für sich selber gesehen wahr sind. Auch wenn Rosenzweig mit religiösen Kategorien und Begriffen operiert, geht es ihm in keiner Weise um die Verteidigung eines Dogmas oder einer einzig wahren Lehre, sondern gerade um eine Begründung des Pluralismus - oder vielleicht besser eines Dualismus von Wahrheitsanteilen, die gerade erst in gelebter Praxis als je meine Wahrheit auch zu Gottes Wahrheit wird. Denn mit seinem Wahrheitsschema deutet Rosenzweig nur die beiden monotheistischen Religionen Judentum und Christentum, die ja auch historisch klar mit einander verbunden sind. Als Zwischenbemerkung sei festgehalten, dass es eine interessante und komplexe Aufgabe für eine religionswissenschaftliche Rosenzweig-Lektüre darstellen würde, zu versuchen, ob sich auch unsere multikulturelle und längst nicht mehr auf die beiden abendländischen Monotheismen reduzierte Welt mit Rosenzweigs System deuten liesse. Rosenzweigs Denken besitzt gerade für die an Positionen der post colonial studies orientierten Kulturwissenschaften ein enormes, noch nicht ausgeschöpftes Inspirationspotential.

\section{Judentum und Christentum}

Dieses Potential wird jedoch dadurch eingeschränkt, dass Rosenzweig nur Juden und Christen als „Arbeiter am gleichen Werk“ (SE 462) beschreibt, und dies gerade nicht im Sinn des aufklärerischen Toleranzgedankens, sondern als Theologie einer notwendigen, wechselseitig sich ergänzenden und komplettierenden Konstellation. Er spricht vom „Sternbild der Wahrheit“ (SE 454), eine präzise Metapher für die Mechanik, die er zwischen Judentum und Christentum am Werk sieht. Es ist eben das gleiche „Werk“, eine Art Uhrwerk, dessen eine Zeit für beide Teilnehmer unterschiedlich abläuft. Seit Einsteins spezieller Relativitätstheorie, die nur einige Jahre vor dem Stern der Erlösung formuliert wurde, und deren erstem Grundsatz, nämlich dem Prinzip der Zeitdilatation bewegter Uhren, weiss man, dass eine solche auf den ersten Blick paradoxe Konstruktion durchaus realistisch sein kann. Um im stellaren Bild zu bleiben, besetzen Judentum und Christentum sich unterschiedlich bewegende Positionen in einem gemeinsamen Raum, zu denen sich die jeweilige Zeit relativ verhält. Rosenzweigs Thesen zu Judentum und Christentum, die er bereits im ersten Buch des dritten Teils des Sterns sehr ausführlich referiert und hier 
nochmals bespricht, lassen sich auf den Gegensatz von Statik im Judentum und Dynamik im Christentum verkürzen. Jude ist man immer schon. Man wird als Jude geboren, Jude-Sein ist dem Juden ein „inneres Zuhause“ (SE 440). Aus dieser Zugehörigkeit qua Geburt folgt auch eine grundsätzliche Bedeutung des Kollektivs im Judentum. Das, was den Juden zum Juden macht, liegt vor dem Einzelleben, es ist die Offenbarung an das Volk, es ist das Volk Israel, dem jeder einzelne immer schon durch seine Geburt von einer jüdischen Mutter angehört. Für den Christen ist es nach Rosenzweig gerade umgekehrt. Das Christentum muss in jedem Menschen immer neu plausibilisiert werden. Man ringt sich sein Christentum ab, Man wird also zum Christen, man ist es nicht, ${ }^{289}$ während man Jude eben immer schon ist. Aus diesem Gegensatz erklärt Rosenzweig auch, dass das Christentum gleichsam nach Aussen gehen und missionieren muss. Die Offenbarung muss in die Welt getragen und damit die Christenheit ausgedehnt werden, während das Judentum immer bei sich bleibt und eigentlich raumlos und atemporal ist. Die Offenbarung des Judentums bedarf keiner Verbreitung, weder zeitlich noch räumlich. Die jüdische Zeit ist die zirkulare Zeit des Sterns selbst, während die christliche Zeit die lineare Zeit derjenigen ist, die im Raumschiff auf den Stern zurasen.

Dieses Bild passt zu einer anderen Metapher, die Rosenzweig im letzten Kapitel des Stern benutzt. Die "Rechnung des Christentums" sei die Addition, die des Judentums die Subtraktion. Damit ist auch eine gewisse defensive Haltung und eine Selbstbezüglichkeit des Judentums gemeint. Das Judentum „wird durch Subtraktion bestimmt, durch Verengung, durch Bildung immer neuer Reste“ (SE 450). Dieses Beharren auf dem Inneren, eigenen, zeigt sich an der zentralen Stellung des jüdischen Gesetzes. Dieses ist nicht auf religiöse oder rituelle Gebote und Verbote oder eine Aufzählung von Vorschiften beschränkt. Der Talmud ist eine Sammlung von Diskussionen zu unterschiedlichen alltäglichen, religiösen, ökonomischen, ethischen oder sozialen Fragen, die rabbinische Literatur umfasst das gesamte Spektrum menschlichen Lebens. Da das Judentum jedoch nach dem Jahr 70 n.u.Z. (als die Römer Jerusalem erobern und den Zweiten Tempel zerstören) keine Staatsreligion mehr ist, keine Eigenstaatlichkeit hat und auch keinen Tempel mehr besitzt, wird die Anwendung des Gesetzes in vielerlei Hinsicht zu einer Textpraktik. Das Gesetz ist "fertig und unveränderlich“ (SE 451). Es geht aber nicht ausschliesslich darum, das Gesetz anzuwenden, sondern es möglichst umfassend zu lesen und zu deuten - es zu „lernen“, wie es im Judentum heisst. Und obwohl natürlich Vorschriften etwa zur rituellen Reinheit, zu den Gebeten etc. im observanten Alltag eine wichtige Rolle spielen, sind sie dennoch immer Gegenstand von

${ }^{289}$ Mit diesem Gedanken bezieht sich Rosenzweig auf den von ihm viel gelesenen frühchristlichen Autor und so genannten Kirchenvater Tertullian, der festhielt: „[...] fiunt, non nascuntur Christiani.“ (=Man wird, ist nicht von Geburt an Christ.), Tertullian, Apologeticum. Verteidigung des Christentums, Lateinisch und Deutsch, München 1952, 122 (Kapitel 18,2). 
gelehrten Auslegungsdebatten. Dieses selbstbezügliche Studium spielt eine konstitutive Rolle für das orthodoxe Judentum. Denn die absolute Einlösung und Einhaltung des Gesetzes ist das virtuelle Ziel, das diese Welt von der kommenden, erlösten unterscheidet. Nach Rosenzweig ist es der "gott-, welt- und menschumfassende Vorgang der Erlösung" (SE 457), der sich zwischen jüdischem Mensch und jüdischem Gesetz abspielt.

Im Christentum dagegen fehlt nach Rosenzweig die Einheit von Glauben und Gefühl. Auch die Theologie des Sohn Gottes, also dass Gott in Jesus Christus wirklich Mensch geworden, gestorben und auferstanden ist, stiftet diese Einheit nicht. Denn es ist schwierig, an die Geschichte von Menschwerdung und Auferstehung und damit verbunden an das Versprechen künftiger Erlösung jedes einzelnen in der Gemeinschaft mit Christus ganz zu glauben. „Ob Christus mehr ist als eine Idee - kein Christ kann es wissen“ (SE 461). Diese christliche Skepsis bildet auch den Ursprung des christlichen Antisemitismus. Die Christen sehen am Juden, dass dieser (durch seine Gebürtlichkeit an das Gesetz gebunden) immer schon die potentielle Erlösung mit sich trägt. Der Christ muss ist aber immer erst unterwegs zu dieser Erlösung, er muss für sie arbeiten. Dennoch stellt das Judentum für das Christentum nicht nur eine Provokation oder Beunruhigung dar, sondern auch eine Versicherung, eine Garantie seiner eigenen tatsächliche Wahrheit. Während die christliche Erlösung nicht konkret zu vergegenwärtigen ist, stellen die vor dem Christen stehenden, lebendigen Juden das nicht zu bezweifelnde Faktum der Durchdringung von Leben und Erlösung dar. Im Judentum kann die Erlösung jederzeit, in jedem Augenblick hereinbrechen. Aus christlicher Perspektive liegt dieser Zeitpunkt der Erlösung jedoch am Ende der Zeit. Juden bleiben Juden bis zu diesem eschatologischen Ende der christlichen Heilsgeschichte, nämlich bis alle Völker das Evangelium angenommen haben.

Diese theologischen Thesen zum Verhältnis von Judentum und Christentum sind hier nur sehr verkürzt dargestellt worden. Im hier zur Diskussion stehenden Teil des Sterns bilden sie filmisch gesprochen eine Rückblende auf das erste Buch des dritten Teils, wo sie religionsgeschichtlich sehr ausführlich hergeleitet werde. Im dritten Buch des dritten Teils möchte Rosenzweig damit lediglich seine Theorie von der Teilhaftigkeit und Tatsächlichkeit der Wahrheit belegen.

\section{Schau und Antlitz, Anfang und Ende}

Die menschliche Erfahrung von Wahrheit ist immer eine teilhafte. Doch Rosenzweig gibt sich nicht mit dieser Beschränkung auf das Partikulare zufrieden. Die ganze Wahrheit gibt sich nämlich im mystisch konnotierten Modus der Schau. Die ganze Wahrheit, die auch Gottes Wahrheit ist, kann geschaut werden, und eine solche Schau findet „jenseits des Lebens“ statt (SE 462). Was ist mit diesem „Jenseits des Lebens“ gemeint? In einem alltäglichen Verständnis würde man wohl sagen, dass jenseits des Lebens eben der Tod ist, und ein 
Schauen der ganzen Wahrheit also nur dem möglich ist, der sich in einer Art Himmel befindet, in einem Leben nach dem Tod, das dem wahren und wirklichen Leben gleichkommt, gegen das das irdische Leben nur ein armseliges Vorspiel darstellt. Dieses landläufige Verständnis scheint aber im Stern der Erlösung nicht zuzutreffen.

Im Tor spricht Rosenzweig davon, dass ,in der Welt selber ein Stück Überwelt“ (SE 471) vorhanden sei und dass die Schau dort „verstattet“ sei. Die Sprache dieses letzten Teils ist teilweise dunkel und führt bewusst an die Grenze zur Unverständlichkeit, sie ist mit Archaismen und an mystische Formeln erinnernden Wendungen aufgeladen. So ist denn auch das Partizip „verstattet" ein merkwürdiges Wort. Sowohl das Grammatisch-kritische Wörterbuch von Adelung als auch das Deutsche Wörterbuch von Jacob und Wilhelm Grimm (die klassischen philologischen Nachschlagewerke des Deutschen) sehen im Verb „verstatten“ ein veraltetes Synonym von „gestatten“: „Statt oder Raum zu etwas geben, doch nur im figürlichen Verstande, Freyheit geben, etwas zu thun, gestatten [...]. “290 Doch bei Rosenzweig ist dieses „Statt oder Raum“ geben wohl ausdrücklich nicht im figürlichen, d.h. übertragenen Sinn gemeint. Ganz real geht es ihm um eine Stätte, einen Ort in dieser Welt. Die „umgelautete Form“ „verstattet“ kommt gemäss Grimm nur „vereinzelt" vor, ${ }^{291}$ aber es ist gerade sie, die Rosenzweig benutzt. Die Bedeutung changiert zwischen der Erlaubnis zur Schau und ihrer Verortung. Tatsächlich scheint Rosenzweig mit dieser räumlichen Begrifflichkeit also eine Einfaltung des Transzendentalen in den Innenraum der Welt zu beschreiben. Es gibt gleichsam eine Art Enklave des Jenseits im Diesseits, ein extraterritorialer Punkt, der sich aber innerhalb unseres Raumes befindet. Von diesem Punkt geht die Schau der ganzen Wahrheit aus - es ist der Ort der Wahrheit.

Diese Stätte der Wahrheit wird als Antlitz beschrieben, und zwar als „ein Antlitz gleich dem eigenen“" (SE 471). Dem Wort Antlitz für Gesicht eignet schon zu Rosenzweigs Zeiten ein archaisierender und biblischer Ton. Das Grimmsche Wörterbuch hält fest: „Luther verwendet das wort in der bibel sehr oft [...]. “ Und: „Heute klingt uns antlitz feierlicher und poetischer als angesicht oder gesicht. "292 Man würde nicht vom Gesicht Gottes sprechen, sondern von seinem Antlitz. Das sakrale Pathos des Wortes Antlitz ist bei Rosenzweig gewollt. Denn dieses Antlitz Gottes ist für Rosenzweig die Wahrheit, die uns anblickt und die wir anblicken. Aber wie ist es möglich, Gottes Antlitz zu sehen, physisch wahrzunehmen? Rosenzweig geht davon aus, dass die Erscheinung der göttlichen Gegenwart analog zur Wahrnehmung des menschlichen Gesichts ist. Antlitz heisst das menschliche Gesicht, insofern es als Gottes Prä-

${ }^{290}$ Lemma „verstatten“, in: Johann Christoph Adelung, Grammatisch-kritisches Wörterbuch der hochdeutschen Mundart, München 1811.

${ }^{291}$ Lemma „verstatten“, in: Deutsches Wörterbuch von Jacob und Wilhelm Grimm, 16 Bände in 32 Teilbänden, Leipzig 1854-1961.

${ }^{292}$ Lemma „antlitz“, in: Deutsches Wörterbuch. 
senz sinnlich wahrgenommen wird, was für jedes Gesicht potentiell möglich ist. ${ }^{293}$ In jeder zwischenmenschlichen Begegnung ist also der transzendentale Punkt der Schau möglich. Ihr Ort ist das Antlitz. Es gibt eine Unsicherheit, ob dieser Schluss nun als Mystik gelesen werden muss, das heisst als Beschwörung einer jegliche diskursive Logik sprengenden Fülle intersubjektiver Erfahrung oder als philosophischer Versuch, einen Begriff für die Erfahrung tatsächlicher Wahrheit zu finden. Als Indiz für ein mystisches Verständnis der Rede vom Antlitz könnte dienen, dass Rosenzweig versucht, die Struktur der Gottesschau anhand von Kategorien des menschlichen Körpers, des Gesichts, zu beschreiben. Diese Analogie hat eine Tradition in der jüdischen Mystik, der Kabbala. ${ }^{294}$ Die Verortung des Sterns in einen weitgespannten Zusammenhang von Spekulationen über die "Gestalt der Gottheit" ist aber letztlich ein Versuch zur Authentifizierung der vorgelegten Wahrheitskonzeption anhand der Tradition.

Das philosophische Verständnis von "Antlitz" würde besagen, dass Wahrheit nur aus einem intersubjektiven Prozess zu gewinnen, also kommunikativ verfasst wäre. Wahrheit ist - das würde dieses zweite Verständnis von Antlitz aussagen - nur intersubjektiv „verstattet“. Das Antlitz ist ein Begriff des Denkens, der zwar der unmittelbaren Anschauung entstammt, aber nicht weniger als andere philosophische Termini abstrakte Bedeutung besitzt. Rosenzweig erklärt, dass er „in Bildern“ spricht: „Aber die Bilder sind nicht willkürlich. Es gibt notwendige und zufällige Bilder" (SE 469). Die Metapher des Antlitzes ist deshalb für ihn notwendig, weil es den Teil des menschlichen Körpers darstellt, mit dem der Mensch „mit der Umwelt in Verbindung tritt“ (SE 470) und, so kann man hinzufügen, die Leiblichkeit, die existentielle Tatsächlichkeit des Menschen mit einer sakralen Konnotation auflädt. Im Antlitz wird Wahrheit authentisch, sie bekommt im menschlichen Gesicht einen lebendigen Ort.

Die Authentizität der Wahrheit muss sich in einer alltäglichen Moral bewähren. Du sollst nun ,von Herzen gut sein und einfältig wandeln mit deinem Gott“. Diese simple Kinderregel bezeichnet Rosenzweig als „Letztes“ (SE 471). Die Schlussrhetorik des Stern ist entschieden einer romantischen Ästhetik des Anfangs im Ende geschuldet: Wie Gott selbst der Erste und der Letzte ist, sich jeder Unterscheidung von Anfang und Schluss entzieht, so muss auch Der Stern der Erlösung auf diese romantische Struktur verweisen. Das Ende des Buches muss Anfang bedeuten. Doch: „Und dies Letzte ist nichts Letztes, sondern ein allzeit Nahes, das Nächste; nicht das letzte also, sondern das Erste. Wie schwer ist solch Erstes! Wie schwer ist aller Anfang!" (SE 471). Das Ende des Stern

${ }^{293}$ Vgl. Martin Brasser, „... nur das rechte blitzt“. Das Motiv des Angesichts und des Antlitzes im Stern der Erlösung von Franz Rosenzweig, in: Yehoyoda Amir/Yossi Turner/Martin Brasser (Hg.), Faith, Truth, and Reason. New Perspectives on Franz Rosenzweig's Star of Redemption, Freiburg i.B./München, 125-136.

${ }^{294}$ Vgl. Gershom Scholem, Von der mystischen Gestalt der Gottheit. Studien zu Grundbegriffen der Kabbala, Frankfurt a.M. 1991. 
der Erlösung, sein Letztes, ist also die Erkenntnis, das dieses Letzte ein Anfang sein muss, nämlich ein Anfang, die Wahrheit zu bewähren, und dass dieser Anfang schwer ist. Aus dieser Perspektive betrachtet wird die Differenz zwischen Anfang und Ende aufgehoben. Es ist eine Aufhebung, die sich letztlich auch der Form des Buches mit seiner linearen Struktur entzieht und die vielleicht eine ganz neue Art des Lesens und Schreibens erfordern würde. „Aller Anfang ist schwer!" heisst es in Wilhelm Meisters Lehrjahren von Goethe. Doch: „Das mag in gewissem Sinne wahr sein; allgemeiner aber kann man sagen: aller Anfang ist leicht, und die letzten Stufen werden am schwersten und seltensten erstiegen. “"295

\section{Weiterführende Lektüre}

Hufnagel, C., (1994), Die kultische Gebärde. Kunst, Politik, Religion im Denken Franz Rosenzweigs, Freiburg, Alber.

Wolfson, E. R., (2021), Nomadism, Homelessness and the Homecoming of the Poet, in: "Into Life", Franz Rosenzweig on Knowledge, Aesthetics, and Politics. Supplements to the Journal of Jewish Thought and Philosophy 31 (2021) 281-342; [https://doi.org./10.1163/9789004468559_013]

${ }^{295}$ Johann Wolfgang Goethe, Goethes Werke. Band 8, Hamburg 1950, 36-37. 